\title{
Comportamiento agronómico en fase vegetativa de vitroplantas de plátano hartón (Musa AAB, Simmonds)
}

Agronomic performance in vegetative stage of hartón vitroplants (Musa AAB, Simmonds)

\author{
Ramírez Duarte Luz Angélica ${ }^{1}$, Ladino Botero Diego Felipe ${ }^{1}$ y \\ Carmen Carrillo Nydia ${ }^{2}$ \\ ${ }^{1}$ I.A., Universidad de los Llanos y \\ ${ }^{2}$ I.A., MSc. Docente Universidad de los Llanos \\ ncarmen@unillanos.edu.co
}

Recibido 31 de Mayo 2'17, Aceptado 28 de Agosto 2017

\section{RESUMEN}

Con el fin de estudiar el comportamiento agronómico en fase vegetativa de vitroplantas de plátano hartón para las condiciones del municipio de Granada, en el departamento del Meta, se realizó la evaluación de las variables: altura, diámetro del pseudotallo, número de hojas, tasa de emisión foliar y duración de ciclo vegetativo; en dos experimentos que abarcaron tanto la fase de vivero (aclimatación) como la de campo (sitio definitivo). Se realizó la comparación con plantas provenientes de cormo y se evaluó la estabilidad de los materiales, el porcentaje de plantas con variaciones somaclonales morfológicas e incidencia de Ralstonia solanacearum. Para el primer experimento se estableció un diseño de bloques completos al azar, con un total de tres (3) tratamientos, tres (3) repeticiones y seis (6) submuestras; la unidad experimental correspondió a 30 plantas, para un total de 270 plantas. Los tratamientos consistieron en suelo con cascarilla de arroz quemada más abono orgánico con 10.9\% de calcio (Ca) (T1), $16.9 \%$ de $\mathrm{Ca}$ (T2) y 22\% de Ca (T3), con una proporción 2:2:1 (V/V) de suelo, cascarilla y materia orgánica comercial, respectivamente. En el segundo experimento, se trabajó bajo el mismo diseño, pero con cuatro (4) tratamientos, cuatro (4) repeticiones y seis (6) submuestras. La unidad experimental trabajada fue de 20 plantas establecidas en campo a distancias de $2 \times 2 \mathrm{~m}$, para una 
densidad de 2500 plantas/ha. Los tratamientos 1,2 y 3 del segundo experimento correspondieron a los mismos del experimento anteriormente descrito, y se compararon con un tratamiento control (T4), es decir colinos comerciales. El análisis de varianza y las pruebas de comparación de medias indicaron que no hubo diferencia $(P>0.05)$ en altura, diámetro de pseudotallo y número de hojas emitidas entre los tratamientos durante la fase de vivero; en campo las plantas provenientes de cultivo in vitro presentaron mayor crecimiento en altura y diámetro del pseudotallo $(P<0.05)$, que las plantas provenientes de cormo. La duración de ciclo vegetativo fue mayor $(\mathrm{P}<0.05)$ para el material procedente de micropropagación en aproximadamente 5 semanas, el cual presentó un $2 \%$ de variaciones somaclonales, una reversión al clon dominico del 10.8\% y una incidencia de Ralstonia solanacearum del $0.83 \%$.

Palabras clave: Micropopagación, colino, vivero, siembra, cultivo.

\section{ABSTRACT}

In order to study the agronomic behavior in the vegetative phase of vitroplants of hartón banana for the conditions of the municipality of Granada, in the department of Meta, the evaluation of variables was performed: height, diameter of the pseudostem, number of leaves, leaf emission rate and duration of the vegetative cycle; in two experiments that included both the nursery phase (acclimation) and the field (final site). The comparison was made with plants from corm and the stability of the materials was evaluated, the percentage of plants with morphological somaclonal variations and incidence of Ralstonia solanacearum. For the first experiment a complete random block design was established, with a total of three (3) treatments, three (3) replicates and six (6) subsamples; the experimental unit corresponded to 30 plants, for a total of 270 plants. The treatments consisted of soil with burned husk of rice more organic fertilizer with $10.9 \%$ of calcium (Ca) (T1), $16.9 \%$ of $\mathrm{Ca}(\mathrm{T} 2)$ and $22 \%$ of $\mathrm{Ca}(\mathrm{T} 3)$, with a $2: 2: 1$ (V/V) ratio of soil, husk and commercial organic matter, respectively. In the second experiment, we worked under the same design, but with four (4) treatments, four (4) replicates and six (6) subsamples. The experimental unit worked was 20 plants 
established in the field at distances of $2 \times 2 \mathrm{~m}$, for a density of 2500 plants/ha. The treatments 1, 2 and 3 of the second experiment corresponded to the same ones of the experiment described above, and were compared with a control treatment (T4), that is to say, commercial colines. The analysis of variance and the comparison tests of means indicated that there was no difference $(P>0.05)$ in height, diameter of pseudostem and number of leaves emitted between the treatments during the nursery phase; on field, plants from in vitro culture showed higher growth in height and diameter of the pseudostem $(P<0.05)$, than plants from corm. The duration of the vegetative cycle was greater $(P<0.05)$ for the material from micropropagation in approximately 5 weeks, which presented a $2 \%$ of somaclonal variations, a reversion to the dominican clone of $10.8 \%$ and an incidence of Ralstonia solanacearum of $0.83 \%$.

Keywords: Micropopagation, colino, nursery, sowing, cultivation.

\section{RESUMO}

Para estudar o comportamento agronômico na fase vegetativa das plantações de vitroplantas de banana hartão para as condições do município de Granada, no departamento do Meta, a avaliação das variáveis foi realizada: altura, diâmetro do pseudossurpo, número de folhas, taxa de emissão da folha e duração do ciclo vegetativo; em dois experimentos que incluíram tanto a fase de enfermagem (aclimatação) como o campo (site final). A comparação foi feita com plantas de corm e a estabilidade dos materiais, a porcentagem de plantas com variações somaclonais morfológicas e a incidência de Ralstonia solanacearum foram avaliadas. Para o primeiro experimento, foi estabelecido um design de blocos completamente aleatório, com um total de três (3) tratamentos, três (3) repetições e seis (6) subamostra; a unidade experimental correspondeu a 30 plantas, para um total de 270 plantas. Os tratamentos consistiram em solo com casca de arroz queimado, além de fertilizante orgánico com 10.9\% de calcio ( $\mathrm{Ca}$ ) ( $\mathrm{T} 1$ ), 16.9\% de Ca (T2) e 22\% de calcio (T3), com uma relação 2:2:1 (V/V) de solo, casca e matéria orgânica comercial, respectivamente. En el segundo experimento, se trabaló sob o mesmo projeto, pero com quatro (4) tratamientos, cuatro (4) 
repeticiones y seis (6) submuestras. A unidade experimental trabalhou foram 20 plantas estabelecidas no campo a distâncias de $2 \times 2 \mathrm{~m}$, para uma densidade de 2500 plantas/ha. Os tratamentos 1, 2 e 3 do segundo experimento corresponderam aos mesmos do experimento descrito anteriormente e foram comparados com um tratamento de controle (T4), isto é, colinos comerciais. A análise de variância e os testes de comparação de meias indicaram que não houve diferença $(P>0.05)$ em altura, diâmetro do pseudosserto e número de folhas emitidas entre os tratamentos durante a fase de enfermagem; no campo, plantas de cultivo in vitro apresentaram maior crescimento em altura e diâmetro do pseudotallo $(P<0.05)$, do que as plantas do corm. A duração do ciclo vegetativo foi maior $(P<0.05)$ para o material de micropropagação em aproximadamente 5 semanas, que apresentou $2 \%$ de variações somaclonais, uma reversão ao clone dominicano de $10.8 \%$ e uma incidência de Ralstonia solanacearum de $0.83 \%$.

Palavras-chave: Micropopagação, colino, creche, semeadura, cultivo.

\section{INTRODUCCIÓN}

El plátano es uno de los productos alimenticios más importantes a nivel nacional, al participar con el $6.8 \%$ del total de la producción agrícola, ocupando el quinto lugar después del café, la caña, la papa y las flores. Su consumo per cápita es de $61.9 \mathrm{~kg} / \mathrm{año}$, lo que permite calificarlo como producto básico en la dieta de los colombianos (Espinal et al., 2006). En cuanto a la introducción del plátano en América, se cree que inicialmente fue llevado por los árabes a España y de ahí traídos a América directamente o vía Islas Canarias. Con motivo de la llegada del plátano a Colombia existen dos teorías: Fue traído a la zona del Darién de donde se difundió por toda la costa Pacífica y, por lo Padres Dominicos por el Orinoco y sembrado inicialmente en el municipio de San Martín, en los Llanos Orientales de Colombia (Martínez, 1998).

En un contexto mundial Colombia figura como el segundo productor de plátano después de Uganda, alcanzando en el 2004 una producción de 2'616.717 toneladas y un rendimiento de 7.8 Ton/ha, en comparación con países como Perú 
(12.3 Ton/ha), Venezuela (11.9 Ton/ha) y Sir Lanka (11.7 Ton/ha) es superado significativamente en términos de rendimiento. Según el Ministerio de Agricultura y Desarrollo Rural, (2011), para el año 2009 a nivel nacional existían 335.226 hectáreas cultivadas en plátano, siendo el primer productor, con mayor participación en hectáreas cosechadas el departamento de Antioquia (12.16\%), seguido del Quindío (10.18\%), Tolima (5.06\%), Nariño (4.72\%) y Meta (4.32\%). Si bien el departamento del Meta es el cuarto productor a nivel nacional, es de resaltar que muestra históricamente el mayor nivel de rendimiento del país, puesto que para el año 2004 alcanzó 17.8 ton/ha, con lo cual duplicó el promedio nacional que fue 8.3 ton/ha, lo que demuestra el potencial productivo del departamento. Sin embargo para los años 2008-2010 se presentó una tendencia decreciente debido principalmente al incremento en las medidas de control empleadas para la disminución de la incidencia de Mycosphaerella fijiensis y a la rápida diseminación de Ralstonia solanacearum; dos patógenos que afectaron la rentabilidad del cultivo, obligando a los agricultores a cambiar el modelo de economía campesina con 1 a 5 ha en asocio con otras especies, a un sistema de monocultivo con 3 a 50 ha que se renuevan completamente luego de una cosecha, máximo dos, bajo modalidades de economía agro empresarial (MADR, 2011).

El Departamento del Meta se consolida como la zona productora de plátano con mayor rendimiento a nivel nacional, superando a departamentos como Antioquía y Quindío en 8.3 y 4.9 ton/ha respectivamente; departamentos que sobresalen por su grado de especialización alcanzado en la producción y exportación de banano y plátano con altos niveles de productividad (Espinal et al., 2006). Lo anterior permite considerar el potencial de oferta del Meta de este producto, principalmente el clon hartón, dado que es el material mejor adaptado a esta región. Sin embargo, un problema frecuentemente reportado por productores es la obtención de semilla de calidad que limita el normal desarrollo del cultivo, al convertirse en medio de diseminación de plagas y enfermedades limitantes como el Moko o Ereke (Ralstonia solanacearum). El cultivo in vitro de tejidos vegetales es una técnica mediante la cual, a partir de un segmento de tejido es posible regenerar en condiciones asépticas una gran cantidad de plantas idénticas a la planta madre; lo 
que permite además de la propagación masiva, la producción de material de siembra libres de agentes patogénicos (Curtis y Schnek, 2006). Actualmente existen vitroplantas comerciales de este clon a disposición de los productores, pero poco se ha difundido sobre el comportamiento de este material en las condiciones agroclimáticas de la región del Ariari, principal zona productora en el departamento del Meta.

Dentro de este contexto la evaluación del comportamiento agronómico en fase vegetativa de plantas de plátano hartón provenientes de cultivo in vitro, frente al clon hartón proveniente de propagación asexual tradicional (cormo), así como la incidencia de Ralstonia solanacearum (enfermedad endémica ampliamente distribuida en todas las zonas productoras del Meta) sobre estos dos materiales; se consolidaría como un aporte valioso a la búsqueda de soluciones a los problemas fitosanitarios, principalmente aquellos que se diseminan por medio de material de siembra.

Por lo anterior, este trabajo buscó evaluar la viabilidad del uso de material vegetativo de plátano hartón obtenido por propagación in vitro, identificando su patrón de comportamiento y su respuesta al patógeno Ralstonia solanacearum, y con ello aportar conocimiento básico para la elaboración de investigaciones de mayor complejidad orientadas hacia la búsqueda de soluciones a los problemas sanitarios en el cultivo del plátano, así como la introducción y fomento en el departamento y la región Orinoquia, de materiales de siembra provenientes de micropropagación.

\section{METODOLOGÍA}

Los experimentos de vivero y campo se realizaron en Granada Meta, que se encuentra ubicada a una altura de $332 \mathrm{msnm}$, temperatura media de $24^{\circ} \mathrm{C}$, humedad relativa del $75 \%$ y precipitación media anual de $2.600 \mathrm{~mm}$. La fase de vivero tuvo inicio en Marzo y finalizó en Abril, con el trasplante a campo, que culminó en Diciembre (Figuras 1 y 2). 


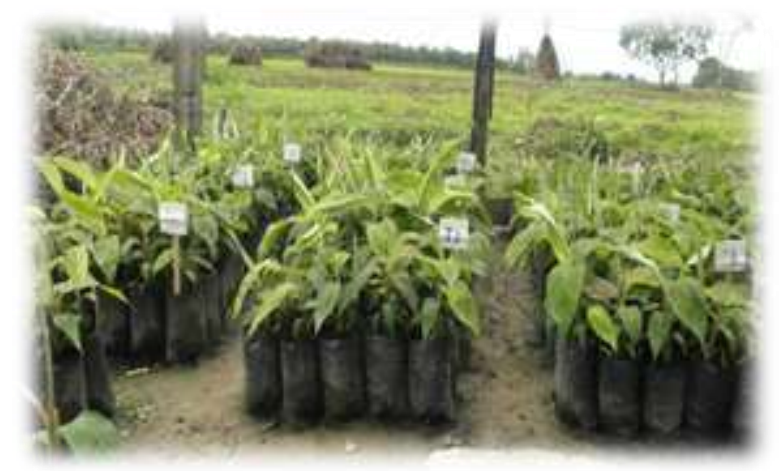

Figura 1. Plátano en fase de vivero

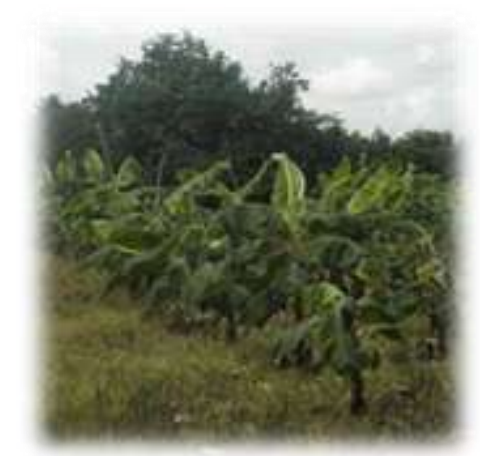

Figura 2. Plátano en fase de campo

Experimento 1. Se utilizaron diferentes fuentes de materia orgánica sobre el desarrollo vegetativo de vitroplantas de plátano hartón en fase de vivero: Se estableció un diseño de bloques completos al azar, con un total de tres (3) tratamientos, tres (3) repeticiones y seis (6) submuestras; la unidad experimental correspondió a 30 plantas, para un total de 270 plantas. Los tratamientos consistieron en suelo con cascarilla de arroz quemada más tres abonos con diferentes niveles de calcio orgánico: $\mathrm{T} 1=10.2 \%, \mathrm{~T} 2=16 \%$ y T3=22\%, mezclados en una proporción 2:2:1 (V/V) de suelo, cascarilla y abono orgánico, respectivamente (Tabla 1$)$.

Tabla 1. Composición química (\%) de los abonos orgánicos

\begin{tabular}{ccccccccc}
\hline $\begin{array}{c}\text { Fertilizante } \\
\text { Orgánico }\end{array}$ & $\mathbf{p H}$ & $\begin{array}{c}\mathbf{N} \\
\text { Total }\end{array}$ & $\mathbf{P}_{2} \mathrm{O}_{5}$ & $\mathrm{~K}_{2} \mathrm{O}$ & $\mathbf{C a O}$ & $\mathbf{M g O}$ & $\mathbf{S O}_{2}$ & Cenizas \\
\hline Ca 10.9\% & 6.85 & 1.1 & 2.3 & 1.8 & 21.4 & 0.73 & 0 & 36.7 \\
$\mathrm{Ca} 16.2 \%$ & 6.98 & 1 & 2.3 & 2.6 & 8.7 & 1.2 & 9.2 & 54.4 \\
Ca 22\% & 7.5 & 1.6 & 2.9 & 3.4 & 4.3 & 0.9 & 47 & 50 \\
\hline
\end{tabular}

La toma de datos se realizó con una periodicidad de 8 días. Las variables evaluadas fueron: porcentaje de sobrevivencia, determinado con base al número de plantas sobrevivientes al trasplante a lo largo del experimento; altura de pseudotallo, tomando la longitud desde el cuello de la plántula hasta el ápice de la hoja más joven abierta; diámetro de pseudotallo, midiendo su grosor a una altura de $3 \mathrm{~cm}$ sobre la superficie del suelo, utilizando un pie de rey (Figura 3); número 
de hojas, determinado mediante marcación de la última hoja emitida (más joven abierta) y contando las hojas que aparecieron después de esta.

Para los ensayos se trabajó con el clon hartón el cual es el más utilizado por los agricultores de la zona por ser el de mejor comportamiento agronómico en las condiciones agroclimatológicas de esta región. Las plantas fueron obtenidas por propagación in vitro, mediante técnica de cultivo de meristemos, previamente endurecidas en sustrato, con una altura entre 8 y $12 \mathrm{~cm}$ y un mes de edad (Figuras 3 y 4 ).

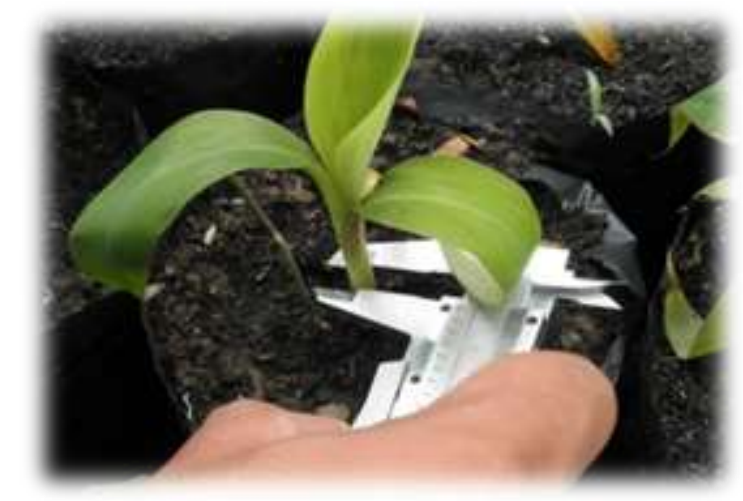

Figura 3. Medida de diámetro de pseudotallo en fase de vivero

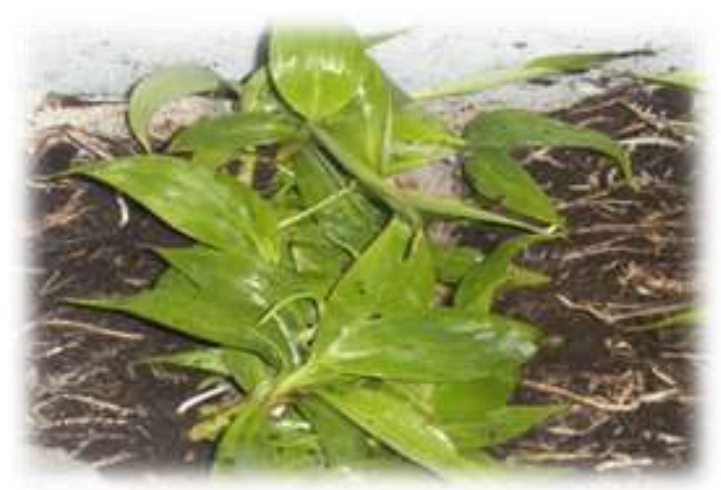

Figura 4. Plántulas de plátano Hartón listas para siembra en bolsas.

En la preparación del sustrato, se utilizó suelo, cascarilla de arroz quemada (98\%) y los abonos orgánicos descritos en la Tabla 1; el sustrato fue tratado con Vanodine en dosis de $1 \mathrm{~L} /$ ha (Figura 5), posteriormente fue empacado en bolsas de polietileno negro perforadas de dimensiones $27 \times 18 \mathrm{~cm}$, en cada una de las cuales fue establecida una plántula (Figura 6).

Previo a la siembra se realizó la medición de la altura y número de hojas a 10 plantas elegidas al azar. Una vez sembrado el material estuvo constantemente durante las primeras seis semanas bajo luz natural al 50\%. La última semana se trabajaron exposiciones directas al sol por periodos de ocho horas como medida de aclimatación y preparación de las plántulas para el trasplante a campo. El plan de fertilización trabajado durante la fase de vivero, se dividió en tres aplicaciones, la primera al momento de la siembra con $5 \mathrm{~g}$ de micorrizas, la segunda a los 20 
días con $5 \mathrm{~g}$ de micorrizas $+10 \mathrm{~g}$ de triple 15, y una tercera fertilización con $15 \mathrm{~g}$ de triple 15 a los 40 días (Figura 7). Para el manejo de plagas, se hizo una aplicación de carbofurano en dosis $1 \mathrm{~L} / \mathrm{ha}$, para control de defoliadores y plagas del suelo.

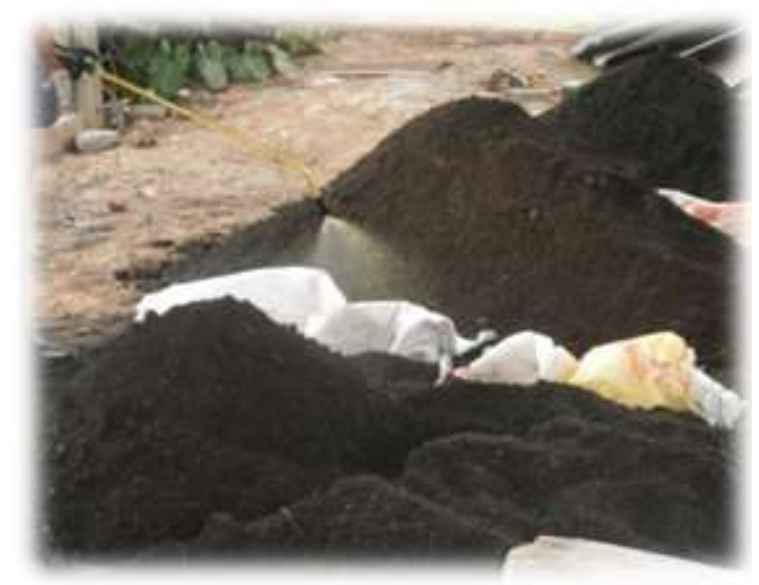

Figura 5. Preparación de sustratos para experimento en vivero

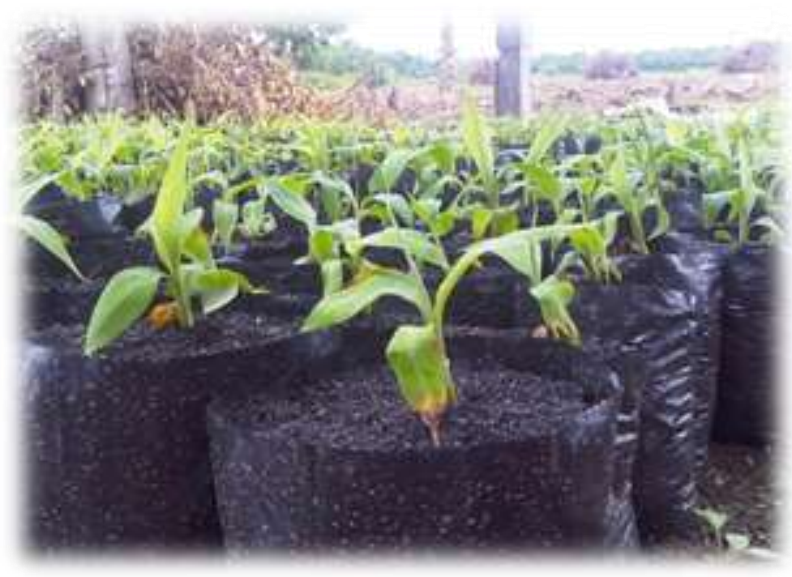

Figura 6. Plántulas en bolsas con sustrato

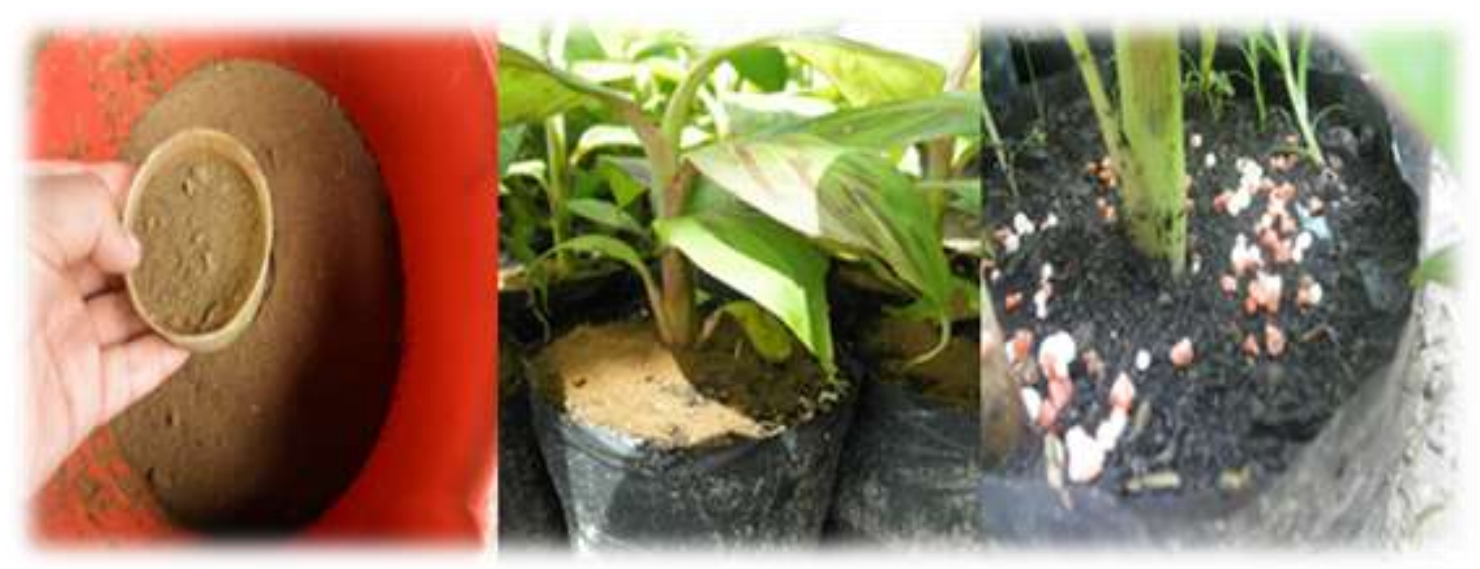

Figura 7. Fertilización en vivero

Experimento 2. Evaluación en campo hasta fase vegetativa de vitroplantas de plátano hartón: En este segundo experimento, se trabajó un diseño en bloques completos al azar, con cuatro (4) tratamientos, cuatro (4) repeticiones y seis (6) submuestras. La unidad experimental trabajada fue de 20 plantas establecidas a distancias de $2 \times 2 \mathrm{~m}$, para una densidad de 2500 plantas/ ha. Los tratamientos T1, T2 y T3 del segundo experimento correspondieron a los mismos del experimento 
anteriormente descrito, comparándose con un tratamiento testigo (T4) colinos comerciales (Figura 8).

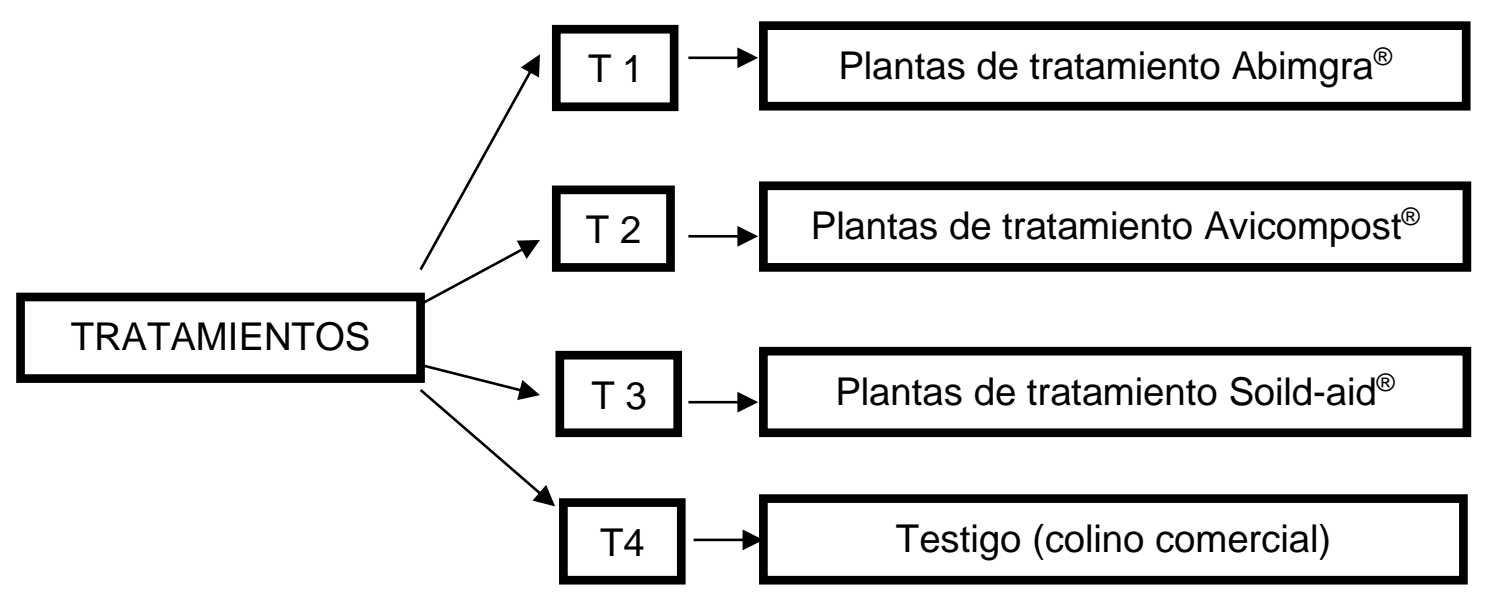

Figura 8. Tratamiento evaluados en fase de campo

Los tratamientos en campo fueron distribuidos al azar. La toma de datos se realizó cada 15 días, a partir de los 30 días después del trasplante a sitio definitivo y hasta la aparición de la bellota. Las variables evaluadas fueron: Porcentaje de supervivencia, estimando el número de plantas que sobrevivieron el trasplante al sitio definitivo; altura de pseudotallo, tomada desde la base del mismo hasta la base del peciolo de la hoja más joven abierta; diámetro del pseudotallo, medido a $10 \mathrm{~cm}$ de la superficie del suelo, con pie de rey (Figura 9); emisión foliar, determinada mediante el conteo repetido en el tiempo del número de hojas emitidas posteriores a la última hoja más joven abierta (hoja 1); marca o identificación en cada evaluación, para lo cual se emplearon fibras, chinches o marcador que se ubican en la base del peciolo de la hoja 1 (Figura 10); duración del ciclo vegetativo, determinando el tiempo transcurrido desde la siembra en campo hasta la aparición de la bellota, incidencia de Ralstonia solanacearum, determinando el porcentaje de plantas afectadas por la bacteria con base en la observación de síntomas externos a lo largo de su ciclo vegetativo; porcentaje de plantas con variaciones somaclonales morfológicas, mediante observación se identificó el número de plantas con patrones fenotípicos diferentes a los descritos para el clon hartón, y se expresó en porcentaje de plantas con variaciones 
somaclonales; estabilidad del material, realizando el conteo de plantas que presentaron reversión a dominico con base en la morfología del racimo, y se determinó el porcentaje de estabilidad genética del material.

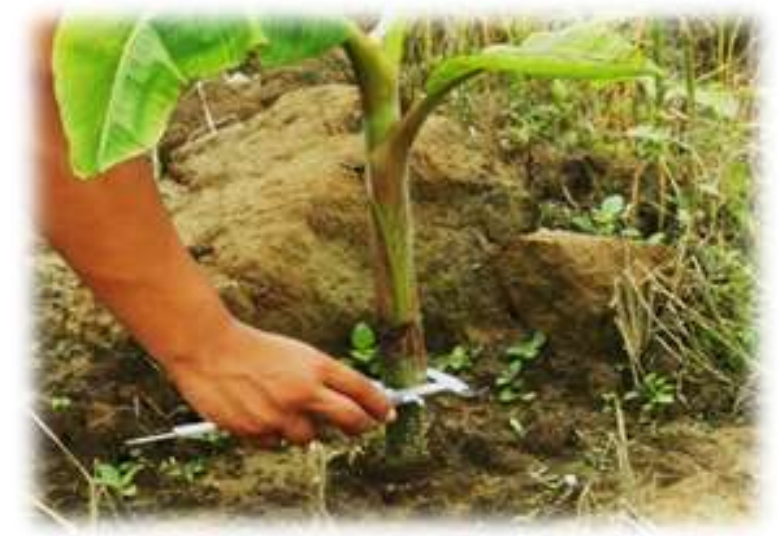

Figura 9. Medición de diámetro de pseudotallo en campo

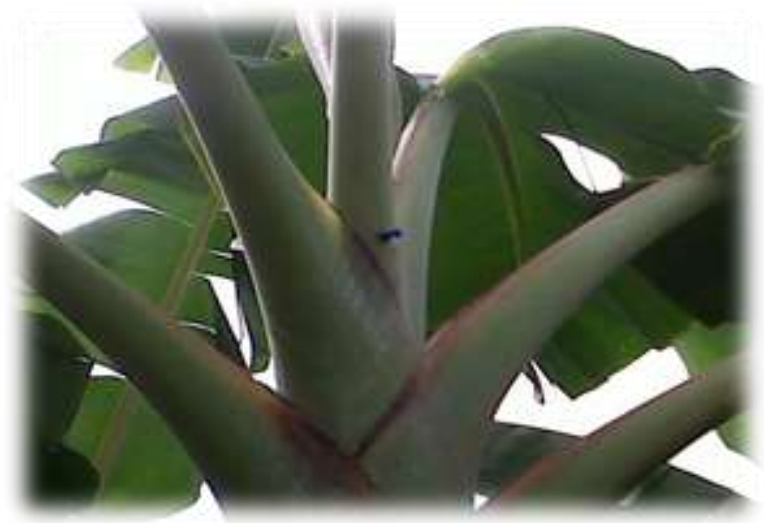

Figura 10. Marcación de hoja número 1 con chinches

La selección de cormos se realizó en un lote de plantas vigorosas, altamente productivas, y con características fenotípicas homogéneas, cuyo peso promedio fue $300 \mathrm{~g}$. El material fue trasplantado a campo luego de haber completado 53 días en vivero, momento en el cual tenían en promedio $76 \mathrm{~cm}$ de altura, $22.8 \mathrm{~mm}$ de diámetro y 8.7 hojas. Las plantas fueron establecidas en huecos de dimensiones $30 \times 30 \times 30 \mathrm{~cm}$, y en el fondo se aplicó materia orgánica junto con una parte de suelo superficial extraído del sitio, y posteriormente se ubicó el cormo recubriéndolo con el suelo restante. El plan de fertilización fue similar para todos los tratamientos, utilizando las siguientes cantidades en gr/ planta: DAP 100 una aplicación; Boro 20 una 30 dos aplicaciones; urea 150 tres aplicaciones. KCL 150 una y 200 dos aplicaciones y 400 gramos de abono con 10.2, 16.9 y $22 \%$ de $\mathrm{Ca}$ orgánico de acuerdo a tratamientos en vivero. El manejo de plagas y enfermedades se realizó mediante labores culturales como el deshoje, deshije y podas, aunque se realizaron algunos controles químicos.

El análisis estadístico, se realizó con el programa SAS versión 9.3, evaluando los datos por medio de análisis de varianza y comparación de medias mediante la prueba de Tukey con un nivel de significancia del 5\%; para determinar la 
existencia de interacción entre el tiempo y los tratamientos se analizaron los datos bajos un arreglo de medidas repetidas en el tiempo, haciendo un análisis multivariado de varianzas (MANOVA) en el cual se trabajó un valor ajustado de $P$, utilizando la prueba de no aditividad de Tukey, con un grado de libertad, con el objetivo de evaluar la interacción entre bloque y tratamiento.

\section{RESULTADOS Y DISCUSIÓN}

\section{Desarrollo vegetativo en fase de vivero}

Porcentaje de adaptación en vivero: La respuesta de las plántulas a la aclimatación en vivero fue buena para T1 y T3 observándose $98.9 \%$ de sobrevivencia, y en el T2 $97.8 \%$ (Tabla 2). Los resultados coinciden con lo reportado por Hwang et al., (1984) y Nava y Villarreal, (1998) quienes mencionan cifras de sobrevivencia superiores al $90 \%$ tanto para el clon hartón como para otros clones, cuando se utilizan las vitroplantas que han crecido en condiciones de laboratorio.

Tabla 2. Sobrevivencia (\%) de vitroplantas en fase de vivero

\begin{tabular}{cccc}
\hline $\begin{array}{c}\text { Semanas después de } \\
\text { trasplante }\end{array}$ & Ca 10.2\%(T1) & Ca 16.9\%(T2) & Ca 22\% (T3) \\
\hline 1 & 100 & 100 & 100 \\
2 & 100 & 98.89 & 100 \\
3 & 100 & 97.78 & 98.89 \\
4 & 98.89 & 97.78 & 98.89 \\
5 & 98.89 & 97.78 & 98.89 \\
6 & 98.89 & 97.78 & 98.89 \\
7 & 98.89 & 97.78 & 98.89 \\
\hline
\end{tabular}

\section{Altura, diámetro, y número de hojas emitidas}

La altura de los tres tratamientos fue similar hasta la cuarta semana, a partir de la cual se empezó a observar diferencias en las medias de esta variable $(P<0.05)$ (Figura 11), este hecho se puede deber a que en las primeras semanas las plantas son sometidas al estrés de adaptarse a un sustrato y condiciones 
diferentes de humedad y temperatura del sitio de donde provienen, lo que hace que su desarrollo sea lento en etapas iniciales. Sin embargo, pese a obtenerse valores de altura final mayor en T1 y T3 que en T2, el ANOVA indica que no existe diferencias $(P>0.05)$ entre los resultados.

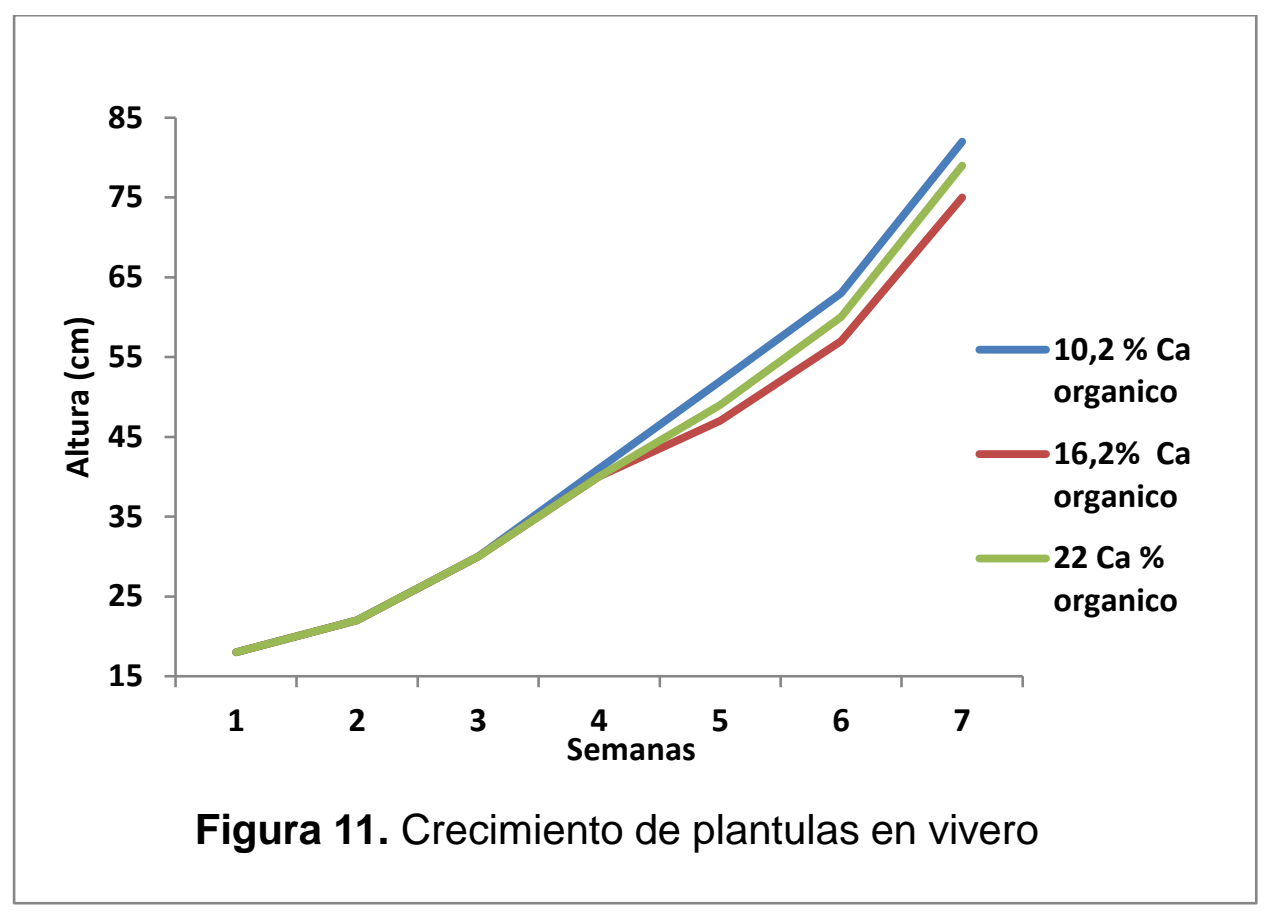

Las variables vegetativas evaluadas no se afectaron con estos tratamientos $(P>0.05)$, dado que las condiciones ambientales fueron iguales, con lo cual se podría inferir que aunque los contenidos nutricionales de los abonos orgánicos son diferentes, estas diferencias no son suficientes para generar estímulos diversos en el desarrollo vegetativo, por lo tanto, se puede recomendar cualquiera de las tres fuentes evaluadas para futuros trabajos de aclimatación de vitroplantas de plátano a nivel de vivero, hasta la séptima semana de edad.

Es importante señalar que la duración de la fase de vivero de este experimento ( 7 semanas) fue mayor al tiempo observado por Nava y Villarreal, (1998) que fue cinco semanas, pero inferior a lo mencionado por autores como Hwang et al., (1984) quienes reportan un periodo de hasta tres meses en fase de vivero. La determinación del momento de trasplante a campo se tomó con base al desarrollo de las plántulas (desarrollo radicular, cantidad y calidad de follaje), el cual a criterio 
de los autores fue adecuado a la séptima semana, pese a esta duración en fase de vivero, que conlleva a una mayor duración del ciclo y costo de producción, el uso de plántulas obtenidas por cultivo de meristemos brinda al agricultor la garantía de llevar a campo un material homogéneo libre de plagas y enfermedades, con el mayor porcentaje de adaptación (100\%) al sitio definitivo, lo cual es difícil de obtener cuando se trabaja con semilla de manera tradicional utilizando cormos.

\section{Fase vegetativa de vitroplantas de plátano hartón en campo}

Porcentaje de sobrevivencia en campo: Los resultados indican que la supervivencia del material proveniente de cultivo in vitro fue del 100\%, puesto que la totalidad de las plantas soportaron el trasplante a sitio definitivo, igual sucedió con el material proveniente de cormo, con una germinación del $100 \%$ y solo un 0.6\% de mortalidad, por la afección por Ralstonia solanacearum. La cifra de sobrevivencia en campo de las plantas micropropagadas es superior a la reportada por Nava y Villarreal, (1998), que fue de $89.7 \%$.

Crecimiento en altura de las plantas: Este parámetro fue similar en T1, T2 y T3 $(P>0.05)$, pero si existe diferencia $(P<0.05)$ de T1 y T3 frente al control $(T 4)$, presentando este último un menor crecimiento en altura del pseudotallo, mientras que el tratamiento T2 mostró igual comportamiento con T4 (Tabla 3). Aunque T1 y T3 al iniciar la fase de campo mostraron promedio de altura similar $(P>0.05)$ a T2 y T4, a partir de la cuarta semana empezaron a disminuir su velocidad de crecimiento en comparación con T1 y T3 (Tabla 3 y Figura 12).

Los resultados del presente trabajo para la variable altura, difieren de lo obtenido por Delgado et al., (2003) y Nava y Villarreal, (1998), quienes no reportan diferencias significativas $(P>0.05)$ para el crecimiento en altura entre material micropropagado y material proveniente de semilla vegetativa (cormo), porque se pudo observar un crecimiento final superior en longitud del pseudotallo por parte de las plántulas provenientes de cultivo in vitro. 


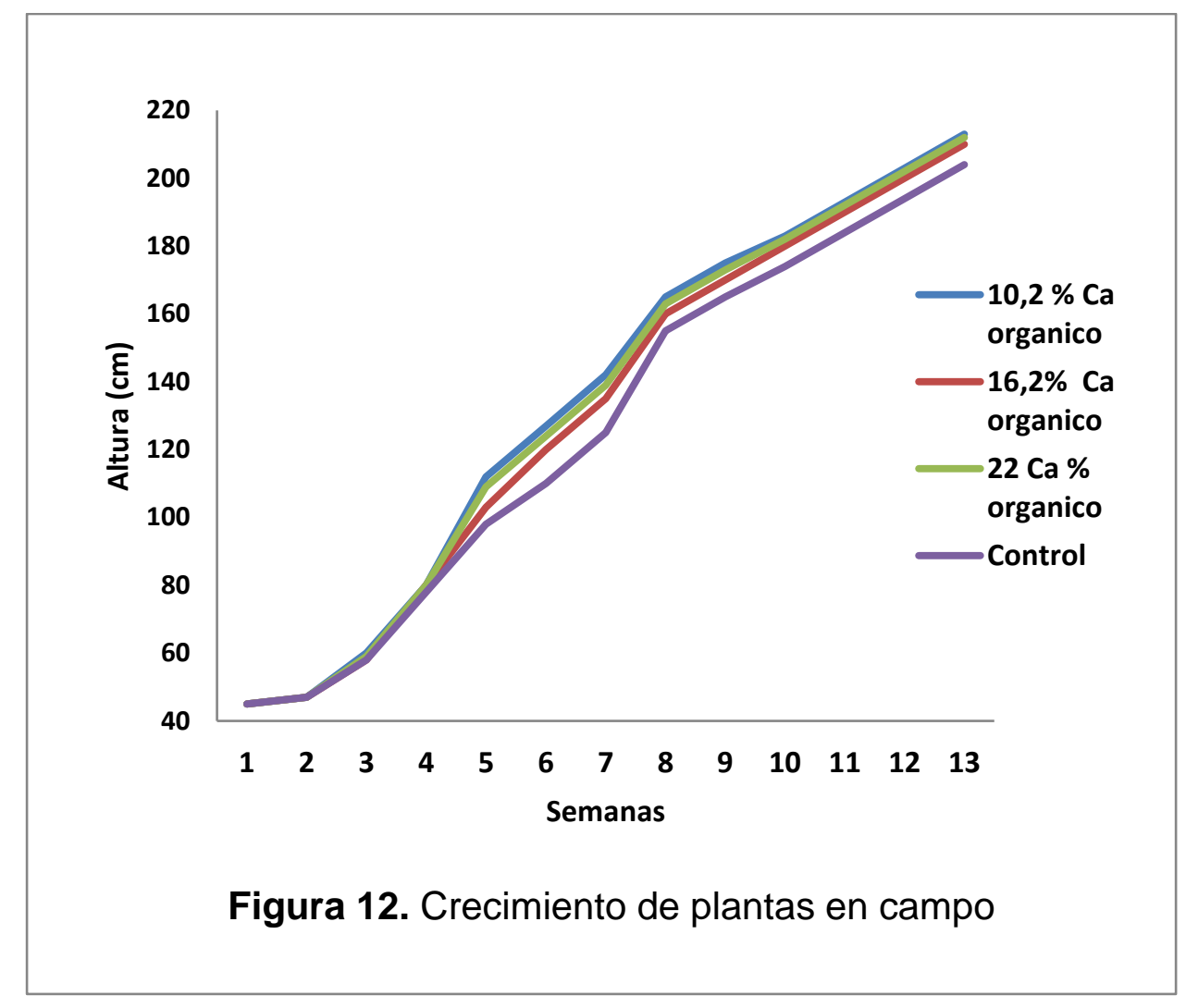

Diámetro de pseudotallo: Para esta variable se encontró que a las 13 semanas del periodo vegetativo del plátano fueron mayores $(\mathrm{P}<0.05)$ los diámetros de los pseudotallos en campo para T1 $(208.56 \mathrm{~mm})$ y T3 $(208.37 \mathrm{~mm})$ con respecto a T4 (201.21 mm), mientras que T2 presentó un grosor similar $(P>0.05)$ a los demás tratamientos (Tabla 3). El comportamiento de la variable diámetro del pseudotallo fue similar al de altura, puesto que inicialmente T2 creció de manera análoga con T1 y T3, pero al final se disminuyó su capacidad de desarrollo, acercándose inclusive un poco más a la media final del control (T4).

De acuerdo con Perez et al., (1997) el vigor es una expresión de altura y circunferencia de las plantas, por lo tanto, se puede afirmar que las plantas provenientes de cultivo in vitro T1 y T3, mostraron mayor vigor $(\mathrm{P}<0.05)$ que aquellas provenientes de cormo (T4). Estos resultados difieren de lo encontrado por Delgado et al., (2003) y Nava y Villarreal, (1998) quienes reportan un mayor crecimiento en plantas provenientes de semilla asexual. Los resultados obtenidos para las variables vegetativas altura y diámetro del pseudotallo, en T1 y T3 son 
satisfactorios al superar en crecimiento a las plantas obtenidas por colino comercial (cormo), lo cual permite esperar racimos de mayor proporción. También se realizó una correlación para ver la inferencia de la diferencia entre las variables iniciales sobre las finales, obteniéndose valores de 0.0879 y 0.1833 para la altura y el diámetro respectivamente, lo cual es muy bajo indicando que los valores iniciales no afectaron los resultados finales en ambas variables.

Emisión foliar en campo: La diferencia en número total de hojas emitidas, entre el control (T4) y los tratamientos T1, T2 y T3, es de acuerdo con el test de Tukey estadísticamente significativa $(P<0.05)$; si se tiene en cuenta que T1, T2 y T3 corresponden a material proveniente de cultivo in vitro, en el cual por las condiciones mismas del método de propagación, las plántulas inician la emisión de hojas desde que inician su proceso de diferenciación, y continúan su emisión durante dos fases previas al trasplante a sitio definitivo (endurecimiento y aclimatación en vivero), este material podría estar produciendo un mayor número total de hojas a lo largo de su ciclo vegetativo.

Tasa de emisión foliar: Las plantas presentaron una emisión foliar media de 1 hoja/semana, siendo similares todos los tratamientos $(P>0.05)$; en acuerdo con Belalcázar, (1991) quien encontró el mismo valor. Se puede afirmar que la tasa de emisión foliar es independiente de la duración del ciclo vegetativo del material y de la cantidad total de hojas emitidas, puesto que a pesar de observar diferencias $(\mathrm{P}<0.05)$ en esta variable para el material proveniente de semilla y el proveniente de cultivo in vitro, la tasa de emisión foliar fue similar $(P>0.05)$ para ambos materiales (Tabla 3).

Duración de ciclo vegetativo: Las plantas de los tratamientos T1, T2 y T3 presentaron un ciclo más largo $(\mathrm{P}<0.05)$ en 4.8 semanas con respecto al control (Tabla 3), resultado que concuerda con lo obtenido por Delgado et al., (2003), debiéndose esta diferencia al proceso de aclimatación (fase de vivero) que requiere todo tipo de material proveniente de cultivos in vitro antes de ser llevado a condiciones de campo, esta información puede ser utilizada por el agricultor para establecer las fechas de siembra conforme a las necesidades del mercado, así 
como también a las condiciones agroclimatológicas, es importante señalar que el periodo vegetativo se puede disminuir en dos semanas, lo cual puede influir positivamente en los costos de producción.

Tabla 3. Variables agronómicas en plantas de plátano en campo durante 13 semanas en respuesta a los tratamientos

\begin{tabular}{ccccc}
\hline Tratamientos & \multicolumn{3}{c}{ Plantas In vitro } & Cormo \\
\hline Variable & Ca 10.9\%(T1) & Ca 16.9\%(T2) & Ca 22\% (T3) & $\begin{array}{c}\text { Colino } \\
\text { comercial (T4) }\end{array}$ \\
\hline Altura (cm) & $213.04^{\mathrm{a}}$ & $210.64^{\mathrm{ab}}$ & $212.43^{\mathrm{a}}$ & $204.11^{\mathrm{b}}$ \\
$\begin{array}{c}\text { Diámetro final } \\
\text { pseudotallo (mm) } \\
\text { Numero de hojas } \\
\text { emitidas }\end{array}$ & $208.46^{\mathrm{a}}$ & $206.67^{\mathrm{ab}}$ & $208.37^{\mathrm{a}}$ & $201.21^{\mathrm{b}}$ \\
$\begin{array}{c}\text { Taaa de emisión } \\
\text { foliar }\end{array}$ & $0.3644^{\mathrm{b}}$ & $0.3608^{\mathrm{b}}$ & $0.3509^{\mathrm{b}}$ & $0.3848^{\mathrm{a}}$ \\
$\begin{array}{c}\text { Duración de ciclo } \\
\text { vegetativo (días) }\end{array}$ & 38.067 & 1.064 & 1.065 & 1.066 \\
\hline abc Superíndices diferentes indica diferencias $(\mathrm{P}<0.05)$ con el test de Tukey
\end{tabular}

Incidencia de Ralstonia solanacearum: A lo largo del desarrollo del experimento en campo solo un $0.06 \%$ de plantas resultaron afectadas por Ralstonia solanacearum, tanto en el material micropropagado como el obtenido por cormo, lo cual confirma que la técnica de propagación por cultivo in vitro no garantiza la tolerancia del material producido, frente a patógenos del medio ambiente, pero sí favorece la sanidad del material, pues la ausencia de la enfermedad en las plantas durante la fase de vivero puede ser un soporte a la garantía de su inocuidad. Por otra parte, aunque el lote elegido para el desarrollo del ensayo no había sido cultivado en plátano por más de 5 años, la ocurrencia de la enfermedad confirma que la bacteria puede permanecer en el suelo, y aún con la rotación de cultivo no se garantiza su erradicación (Tapiero, 2008), a lo anterior se le suma la amplia gama de especies arvenses hospederos de $R$. solanacearum.

Variaciones somaclonales morfológicas y estabilidad del material: Sandoval et al., (1991) y Nava y Villarreal, (1998) han reportado cambios fenotípicos en materiales de plátano, micropropagados y obtenidos por semilla vegetativa 0 
cormo, los cuales se denominan variaciones somaclonales, cuya alta ocurrencia se consolida como una de las grandes desventajas de los materiales obtenidos por técnicas de cultivo in vitro. Los resultados del presente trabajo arrojan un $1.66 \%$ de plantas con estas variaciones como enanismo (Figura 13), coloración morada en pseudotallo y peciolos, y/o filotaxia inusual (Figura 14), se considera que es un porcentaje bajo si se compara con lo obtenido por Nava y Villarreal, (1998). En el momento de la formación del racimo se cuantificó las plantas que presentaron un proceso de reversión a dominico de $10.83 \%$, esto determinado por las características morfológicas del racimo (Figura 15); de esta manera se obtuvo un $10.83 \%$ de plantas con racimo fuera de tipo, siendo inferior al $23.4 \%$ que fue lo obtenido por Nava y Villarreal, (1998) pero superior al $2.5 \%$ observado por Vuylstake et al., (1991) quienes trabajaron con plantas del clon falso hartón. Este tipo de variaciones son mayores en plantas obtenidas por micropropagación en comparación a las propagadas por cormo (Sandoval et al., 1991). Sin embargo, es posible que parte de la causa del porcentaje de reversión encontrado pueda ser debido a la procedencia del material trabajado (clon hartón procedente del Urabá Antioqueño), por lo cual para futuros estudios se recomienda trabajar con vitroplantas obtenidas a partir de material de la región.

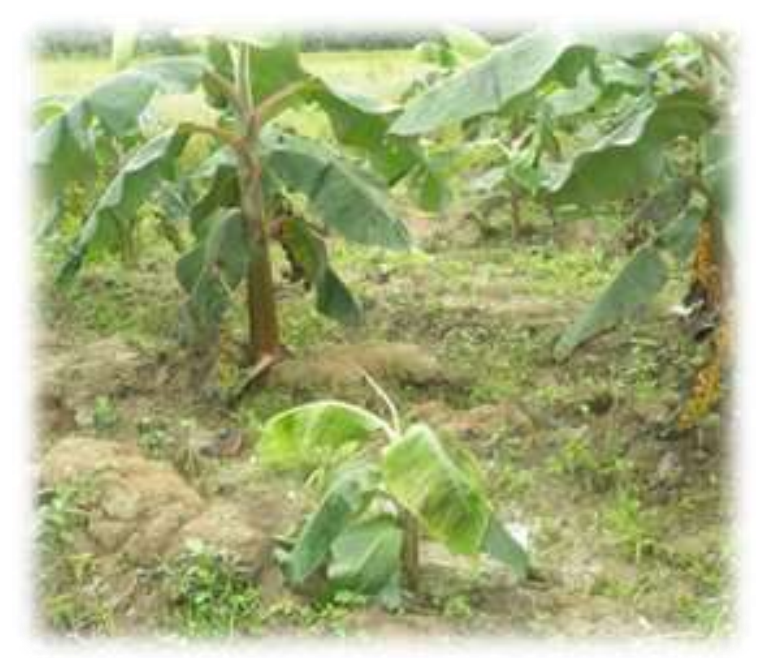

Figura 13. Planta enana

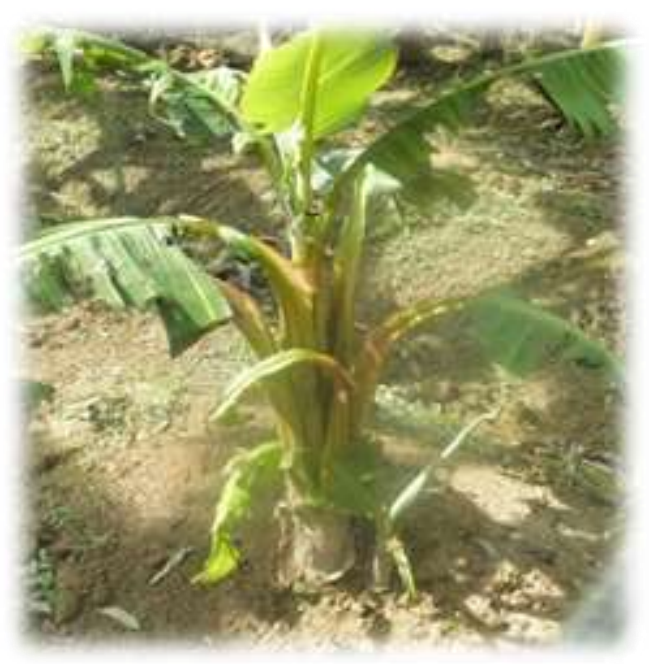

Figura 14. Planta de seis meses con filotaxia inusual y enanismo 
El fenómeno de la reversión puede consolidarse como una desventaja para el uso a nivel comercial de este material, puesto que no se puede garantizar un racimo de características deseables proveniente de estas plantas fuera de tipo, plantas que desde un inicio tienen un costo bastante elevado si se compara con el costo de un colino o cormo comercial.

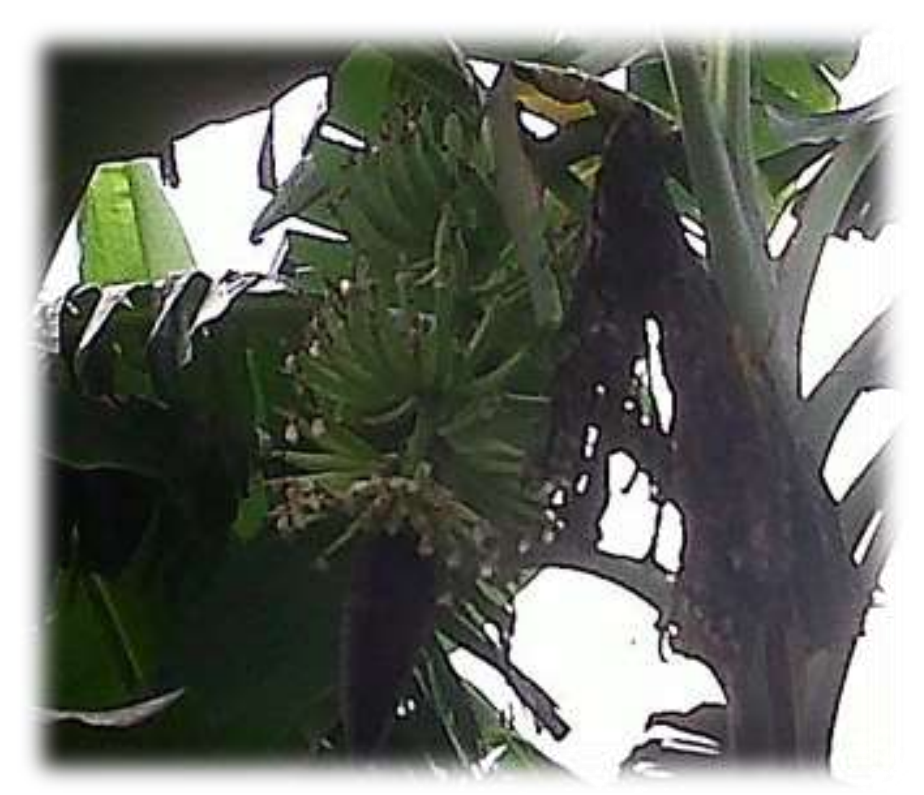

Figura 15. Racimo fuera de tipo, reversión a Dominico

Los resultados de este trabajo permiten afirmar que el comportamiento agronómico hasta fase vegetativa de plantas de plátano hartón procedente de cultivo in vitro, bajo las condiciones del municipio de Granada Meta, es satisfactorio, presentando un mayor vigor (expresado en altura y diámetro del pseudotallo) que plantas procedentes de cormo. Vale la pena resaltar que el $100 \%$ de las vitroplantas llevadas a campo fueron plantas sanas, lo cual es de gran importancia porque en la zona de trabajo se ha encontrado en los predios alta incidencia de Ralstonia solanacearum, y su difícil erradicación hace necesario la búsqueda de medidas mitiguen su diseminación; el uso de vitroplantas si bien no es garantía de inocuidad a lo largo del cultivo, sí puede brindar la seguridad de tener un material de siembra inicial libre de patógenos. 
Por otro lado, pese al conocimiento de la existencia de este material, los agricultores no confían en su calidad para su uso, no solo por desconocimiento de su comportamiento sino también por su alto precio. Ahora bien, si se tiene en cuenta que para el uso del material micropropagado es indispensable pasar por la etapa de vivero, que puede incrementar sus costos de producción y homogeneidad del cultivo, el corto ciclo que va desde trasplante a sitio definitivo hasta la floración, puede compensar este incremento al disminuir los costos de manejo de dos semanas (deshoje, aplicaciones químicas para control de Mycosphaerella fijiensis y jornales de cosecha). También permite seleccionar aquellas plantas de características no deseables y descartarlas antes de su trasplante a sitio definitivo, logrando así que este material sea homogéneo, con buen vigor y libre de patógenos, como lo fue para el presente ensayo en el que se obtuvo un $100 \%$ de sobrevivencia y material libre de patógenos.

Finalmente, la reversión al clon dominico constituye una desventaja al comprometer la homogeneidad y calidad de la producción, fenómeno que aunque se presenta de manera natural en plantas propagadas por cormo comercial, el porcentaje de presentación es mayor cuando las plantas son obtenidas por cultivo in vitro. Otro factor que puede constituirse como una desventaja del uso de este material a nivel comercial son las variaciones somaclonales como el enanismo, la filotaxia inusual, la variación en el color de pseudotallo y peciolos, deformaciones en racimo, entre otras, porque pueden representar pérdida de plantas en cualquier edad, y de esta manera afectar la producción final.

\section{CONCLUSIONES}

Se encontró que los tratamientos aplicados en este experimento, no presentaron diferencias sobre el crecimiento de las plántulas, lo que permite inferir que el uso de cualquiera de los tres abonos orgánicos en cultivos arrojará resultados similares. Así mismo, el comportamiento agronómico de plantas propagadas por cultivo in vitro frente a las propagadas por cormo o colino comercial, en términos generales es satisfactorio, en la medida en que se obtienen plantas a floración con mayor vigor. 
Bajo las condiciones del experimento el material obtenido por cultivo in vitro, presentó características favorables de sobrevivencia y adaptación tanto en condiciones de vivero como en campo, en esta última fase mostró un mayor vigor expresado en una mayor altura y diámetro del pseudotallo frente al control.

Las plántulas procedentes de micropropagación in vitro presentaron un ciclo vegetativo más largo que plantas provenientes de cormo, y mayor número total de hojas emitidas; sin embargo, la tasa de emisión foliar no se vio afectada por esto, puesto la misma en los dos materiales no presentó diferencias.

Por otra parte la presencia de Ralstonia solanacearum sobre plantas micropropagadas confirma la susceptibilidad de este material al llegar a condiciones de campo, es decir, que la técnica de propagación por cultivo in vitro, no garantiza que el material sea tolerante a patógenos del ambiente.

\section{REFERENCIAS BIBLIOGRÁFICAS}

1. Belalcázar S.L. El cultivo del plátano (Musa AAB Simmonds) en el trópico. Instituto Colombiano Agropecuario, Armenia, Colombia. 376 p. 1991.

2. Curtis H., Schnek A. Invitación a la Biología. Ed. Médica Panamericana, Madrid, España. 600 p. 2006.

3. Delgado E., González O., Romero D., Moreno N. Comportamiento productivo de vitroplantas del clon plátano Hartón (Musa $A A B$ ) en los llanos occidentales de Venezuela. Revista de la Facultad de Agronomía. 29 285-294. 2003.

4. Espinal C.F., Martínez H.J., Peña Y. La cadena del plátano en Colombia. Una mirada global de su estructura y dinámica 1991-2005. Ministerio de Agricultura y Desarrollo Rural (MADR). Observatorio Agrocadenas Colombia, Bogotá, Colombia. 38 p. 2006.

5. Hwang S., Chen C., Lin J., Lin H. Cultivation of banana using plantlets from meristem cultures. HortScience. 19 (2): 231-233. 1984.

6. MADR, Ministerio de Agricultura y Desarrollo Rural. Cadena de Plátano. MinAgricultura, Bogotá, Colombia. 28 p. 2011.

7. Martínez A. El cultivo del plátano en los llanos orientales. Corporación Colombiana de Investigación Agropecuaria-CORPOICA. Editorial Siglo XX, Villavicencio, Colombia. 59 p. 1998.

8. Nava C., Villarreal E. Efecto de los métodos de transporte y aclimatación sobre las características y rendimiento de las plantas de plátano Hartón (Musa AAB) originadas por cultivos de tejidos. Revista de la Facultad de Agronomía. 15 (2): 115-122. 1998.

9. Perez L., Acuña P., Sandoval J. Evaluación agronómica de los tetraploides FHIA 01 y FHIA 02. Corbana. 22 (47): 11-19. 1997. 
10. Sandoval J.A., Tapia A.C., Müller L.E., Villalobos V.M. Observaciones sobre la variabilidad encontrada en plantas micropropagadas de Musa cv. Falso Cuerno AAB. Observations on the variability in micropropagated plants of plantain cv. False Horn AAB. Fruits (Paris). 46 (5): 533-539. 1991.

11. Tapiero A.L. Epidemiología y manejo de enfermedades de cultivo de plátano en el piedemonte Ilanero. Corporación Colombiana de Investigaciones Agropecuaria, Villavicencio, Colombia. 40 p. 2008.

12. Vuylstake D., Swennen R., De Langhe E. Somaclonal variation in plantains (Musa spp. AAB group) derived from shoot-tip culture. Fruits (Francia). 46 (4): 429-439. 1991. 